\title{
Counterpart candidates to the unidentified Fermi source OFGL J1848.6-0138
}

\author{
P. L. Luque-Escamilla ${ }^{1}$, J. Martí2 , A. J. Muñoz-Arjonilla ${ }^{2}$, J. R. Sánchez-Sutil ${ }^{2}$, J. A. Combi ${ }^{2,3}$, and E. Sánchez-Ayaso ${ }^{2}$ \\ 1 Dpto. de Ing. Mecánica y Minera. EPSJ, Universidad de Jaén, Campus Las Lagunillas s/n, Edil. A3. 23071 Jaén, Spain \\ e-mail: peter@ujaen.es \\ 2 Departamento de Física. EPSJ. Universidad de Jaén. Campus Las Lagunillas s/n. Edif. A3, 23071 Jaén. Spain \\ e-mail: [jmarti;ajmunoz; jrssutil; jcombi; esayaso]@ujaen.es \\ 3 Facultad de Ciencias Astronómicas y Geofísicas. Universidad Nacional de La Plata. Paseo del Bosque. B1900FWA La Plata. \\ Argentina
}

Received 17 April 2009 / Accepted 8 September 2009

\begin{abstract}
Aims. We aim to contribute to the identification of the counterpart for one of the bright sources of gamma-rays in the catalogue obtained and released by the Fermi collaboration.

Methods. Our work is based on a extensive identification of sources from different wavelength catalogues and databases.

Results. As a first result. we report the finding of a few counterpart candidates inside the 95\% confidence error box of the Fermi LAT unidentified gamma-ray source OFGL J1848.6-0138. The globular cluster GLIMPSE-C01 is remarkably distinctive being among the most peculiar objects consistent with both the position uncertainty in the gamma-ray source and a conceivable physical scenario for gamma-ray production. The Fermi-observed spectrum is compared with theoretical predictions in the literature and the association is found to be plausible but not yet certain because of its low X-ray to gamma-ray luminosity ratio. Other competing counterparts are also discussed. In particular, we pay special attention to a possible Pulsar Wind Nebula inside the Fermi error box, whose nature is yet to be confirmed.

Conclusions. Both a globular cluster and an infrared source resembling a Pulsar Wind Nebula were found to be in positional agreement with OFGL J1848.6-0138. In addition, other interesting objects in the field are also reported. Future gamma-ray observations will reduce the position uncertainty and we hope eventually confirm one of the counterpart candidates reported here. If GLIMPSE-C01 is confirmed together with the possible Fermi detection of the well known globular cluster 47 Tuc, then this would provide strong support to theoretical predictions that globular clusters are possible gamma-ray sources.
\end{abstract}

Key words. globular clusters: general - globular clusters: individual: 47 Tuc - gamma rays: observations - stars: winds, outflows globular clusters: individual: GLIMPSE-C01

\section{Introduction}

The collaboration operating the Fermi Large Area Telescope (LAT) has released a first catalogue of highly-significant gamma-ray sources based on their first three months of observation (Abdo et al. 2009a). The LAT instrument onboard Fermi is extensively described in Atwood et al. (2009) and references therein. Its performance represents a significant step forward with respect to previous gamma-ray space missions, such as the COMPTON-GRO satellite, whose poor angular resolution rendered very difficult the identification of most sources. Among the 205 Fermi bright sources reported so far with significance of $10-\sigma$ or higher, 38 of them remain unassociated with any known object at lower energies.

We have carried out a cross-identification search of these unidentified Fermi sources with different catalogues and databases. The typical $95 \%$ confidence error radius of bright Fermi sources is within 10 to 20 arcmin. Despite the remarkable improvement compared to past missions, it is not unusual to find several counterpart candidates consistent with Fermi error circles. However, on a few occasions we do find one or a few potentially interesting objects that could be responsible for the gamma-ray detection. One of these cases corresponds to the Fermi source OFGL J1848.6-0138, whose error box contains the globular cluster GLIMPSE-C01 (Kobulnicky et al. 2005) among other possible counterparts.

In this paper, we first devote our attention to the evidence in support of a globular cluster (GC) association both from the observational and theoretical point of view. The possibility of GCs as a new class of gamma-ray sources was predicted many years ago by different authors (Chen 1991; Tavani 1993). The production of gamma-ray photons is expected to be powered by a population of millisecond radio pulsars (MSPs) inside the $\mathrm{GC}$, estimated to be of the order of $\sim 10-10^{2}$. These pulsars continuously inject relativistic leptons into the GC medium either from their inner magnetospheres or accelerated in the shocks created by the collision of individual pulsar winds. Theoretical predictions assessing the chances of their detection by the new generation of Cherenkov and satellite gamma-ray telescopes assume that the gamma-ray emission is produced by inverse Compton scattering of these leptons with the stellar and microwave background radiation (Bednarek \& Sitarek 2007). The feasibility of this physical scenario is further enhanced by the suggested identification of the well known GC NGC 104 (47 Tuc) with one of the Fermi gamma-ray sources, i.e., OFGL J0025.1-7202 (Abdo et al. 2009a).

Secondly, we report alternative counterpart candidates inside the OFGL J0025.1-7202 error circle whose nature cannot yet be 


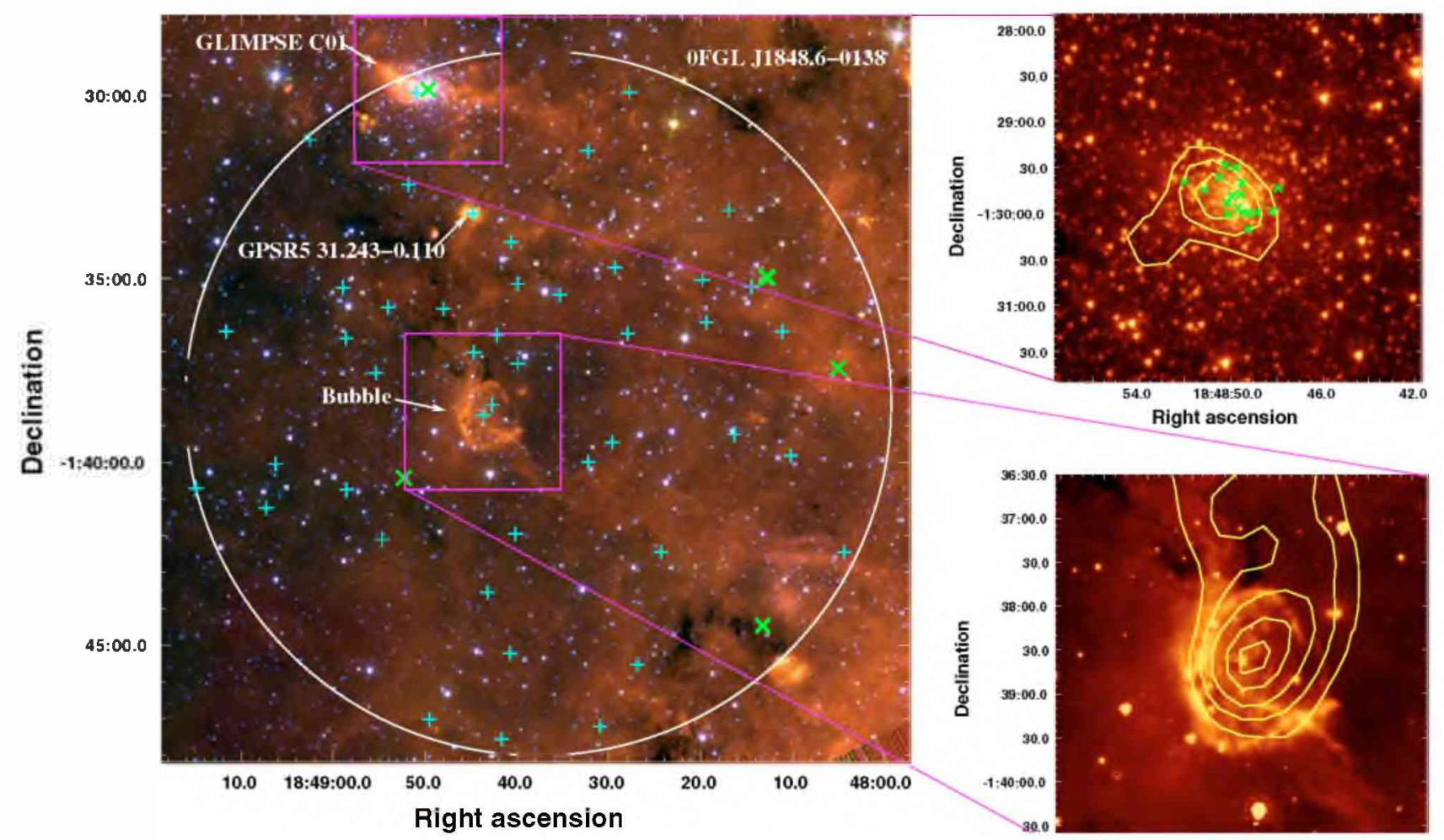

Fig. 1. Left. Tri-colour GLIMPSE image covering the $95 \%$ confidence position of the gamma-ray source 0FGL J1848.6-0138 shown as a white circle. Blue crosses represent radio sources in the field from the NVSS catalogue and green crosses mark the location of X-ray sources detected by XMM-Newton. Right. The right panels illustrate an enlarged view of both the GC (3.6 $\mu \mathrm{m}$, top) and the bubble-like object ( $8 \mu \mathrm{m}$. bottom). including their respective NVSS radio emission as yellow contours with angular resolution of $45^{\prime \prime}$. The emission levels shown correspond to 3.4 . and 5 times the local rms noise of $1 \mathrm{mJy}$ for the GC and 3.9.18.30, and 40 times for the apparent bubble source. Small green crosses are Chandra $\mathrm{X}$-ray sources. GLIMPSE-C01 appears as a faint radio source and contains numerous X-ray sources detected by Chandra marked as small green crosses. On the other hand, the proposed bubble is a strong radio emitter and its possible nature is discussed in the text.

fully established. It is interesting that one of them could be a pulsar wind nebula (PWN). The association of gamma-ray sources with these late products of stellar evolution is well established and the Crab nebula is the most prototypical example. Whether a PWN or a less conventional kind of counterpart, such as a GC, is behind OFGL J0025.1-7202 is an issue yet to be resolved.

\section{Cross-identification of Fermi and multiwavelength archival data}

We initially performed a quick cross-correlation of unidentified Fermi sources with different radio, infrared, and X-ray catalogues and databases, such as the NRAO Very Large Sky Survey (Condon et al. 1998), hereafter NVSS, the Spitzer/IRAC GLIMPSE Survey (Benjamin et al. 2003), and the XMMNewton Serendipitous Source Catalog, 2nd Version, $2 \mathrm{XMM}^{1}$, respectively.

As a result, the case of OFGL J1848.6-0138 is remarkable because of the obvious presence of the GC GLIMPSEC01 $\left(l=31^{\circ}: 3, b=-0.1\right)$ inside its Fermi 9:6 radius of $95 \%$ confidence. In the left panel of Fig. 1, we show the composite $(3.6,5.8$, and $8.0 \mu \mathrm{m}$ bands) GLIMPSE image of the field where the GC is clearly detected. We also find another interesting sources consistent with the OFGL J1848.6-0138 position. Among them, there is the ultracompact HII region GPSR5 31.243-0.110 and an apparent bubble previously unreported located at RA $=18^{\mathrm{h}} 48^{\mathrm{m}} 43^{\mathrm{s}}$ and Dec $=-01^{\circ} 38^{\prime} .7$.

\footnotetext{
http://heasarc.gsfc.nasa.gov/FTP/xmm/data/ catalogues/2XMMcatv1.0.fits.gz
}

Both the GC and the bubble are also detected at radio wavelengths (see right panel of Fig. 1). GLIMPSE-C01 appears as a faint source and contains numerous X-ray emitters detected by Chandra. On the other hand, the proposed bubble is a strong radio source and its possible nature will be discussed below. Its morphology is reminiscent of a PWN, but we remain unable to classify it as explained in the following discussion.

\section{Discussion}

In this section we assess the different possible counterparts reported in this paper.

\subsection{The GC GLIMPSE-C01 as a candidate counterpart}

This heavily obscured $\left(A_{V}=15 \pm 3\right.$ ) cluster was originally reported and studied in detail a few years ago by Kobulnicky et al. (2005). It appears to have an estimated mass of at least $\sim 10^{5} M_{\odot}$ and an age of a few gigayears. The distance to GLIMPSE-C01 is still highly uncertain and values in the range 3 to $5 \mathrm{kpc}$ have been proposed.

Both radio and X-ray emission coincident with this GC has been also reported by different authors (Kobulnicky et al. 2005; Pooley et al. 2007). The marginal and extended radio detection comes from the NVSS survey with an integrated flux density of $20.5 \pm 3.6 \mathrm{mJy}$ at $20 \mathrm{~cm}$. Inspection of the Very Large Array (VLA) archive identifies data sets at the GC position obtained in 1990 at the same wavelength but using the B array configuration, which are of higher angular resolution than the NVSS. We 


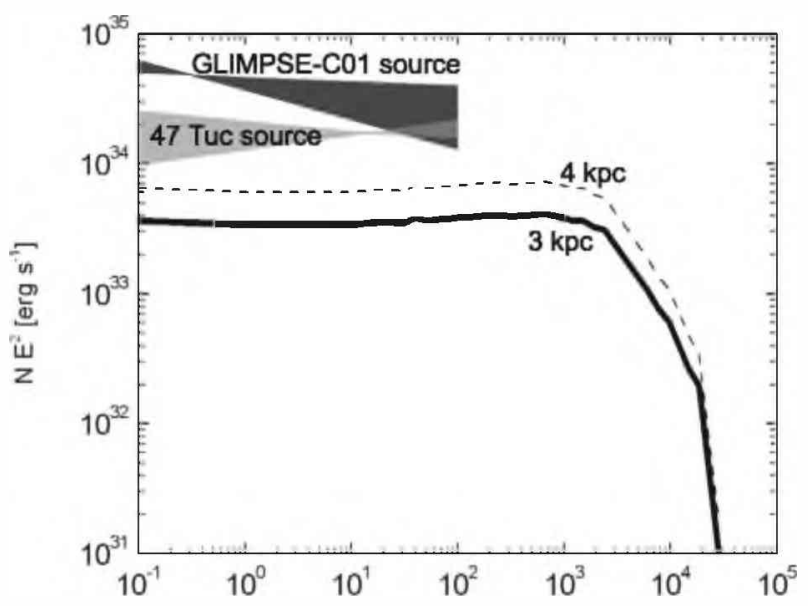

Fig. 2. Comparison of the observed Fermi emission for OFGL J1848.6-0138 and OFGL J0025.1-7202 in the GLIMPSE C-01 and 47 Tuc globular cluster fields. respectively, with some of the gammaray predictions discussed in the text (Bednarek \& Sitarek 2007). The shaded regions correspond to the spectral fit uncertainty and reasonable distances to both clusters of 3 and $4 \mathrm{kpc}$ are assumed.

recalibrated them to produce a radio map of high angular resolution. As a result, no compact radio sources were detected above four times the RMS noise of $0.25 \mathrm{mJy}$ beam ${ }^{-1}$. This implies that the radio emission is intrinsically extended or is produced by the combined effect of faint point-like radio sources.

The X-ray emission observed with the Chandra satellite (Heinke et al. 2005; Pooley et al. 2007) is resolved well into both many point-like sources inside the GC radius and a diffuse component. These objects are most probably a mixture of cataclysmic variables, quiescent low-mass X-ray binaries (LMXB), and MSPs, among other objects. The intrinsic total X-ray luminosity of the GC in the $0.5-8 \mathrm{keV}$ band is estimated to be $\sim 2 \times 10^{33} \mathrm{erg} \mathrm{s}^{-1}$.

The finding of a GC consistent with a bright Fermi source is remarkable and deserves careful attention. Beyond the positional coincidence, the key issue in claiming a possible association is the availability or not of a physical scenario consistent with the observed gamma-ray flux. As quoted in Sect. 1, expectations of the gamma-ray emission from GCs are available in the literature (Bednarek \& Sitarek 2007). The key model parameters are the spectral index of the power-law energy distribution for the leptons injected by the MSP population $(\alpha)$, the GC stellar luminosity $(L)$, the lepton energy cutoffs, the energy conversion efficiency $(\eta \simeq 0.01$ ), the pulsar surface magnetic field (usually $B=10^{9} \mathrm{G}$ ), and the spin period (usually a few $\mathrm{ms}$ ). The magnetic field inside the GC is fixed to $10^{-6} \mathrm{G}$ and their adopted number of MSPs is $N_{\mathrm{p}} \simeq 100$.

In Fig. 2, we plot the theoretical predictions and the observed spectrum for the two Fermi sources i.e., OFGL J1848.6-0138 in discussion here and the similar OFGL J0025.1-7202. The latter is likely to be related to the GC 47 Tuc specifically modelled by Bednarek \& Sitarek (2007). Given that it seems reasonable to initially assume that a similar emission mechanism could be at work in both clusters GLIMPSE-C01 and 47 Tuc, we scaled the same model to their conceivable distances of 3 and $4 \mathrm{kpc}$. The 0FGL J1848.6-0138 spectrum can be represented by $N\left(\right.$ ph erg $\left.{ }^{-1} \mathrm{~cm}^{-2} \mathrm{~s}^{-1}\right)=2.40 \times 10^{-8}[E / \mathrm{GeV}]^{-2.14}$. This is simply the result of fitting a simple power-law spectrum to the Fermi gamma-ray flux measurements in the $0.1-1 \mathrm{GeV}$ and $1-100 \mathrm{GeV}$ bands (Abdo et al. 2009a). The lepton energy limits are between 1 and $3 \times 10^{4} \mathrm{GeV}$. A similar procedure was followed for OFGL J0025.1-7202. Based on the available Fermi fluxes, it seems that the parameters $\alpha=2, L=7.5 \times 10^{5} L_{\odot}$, and a low energy cutoff $E_{\min }=1 \mathrm{GeV}$ provides the theoretical prediction in closest agreement with observations, although both Fermi spectra appear to significantly exceed the model.

In this qualitative comparison the non-perfect agreement may be due to several different effects not correctly taken into account. For instance, the contribution to the gamma-ray spectrum at low energies by the scattering of the microwave background radiation could not be negligible in the case of GLIMPSE-C01, whose stellar luminosity $\left(L \simeq 10^{5} L_{\odot}\right)$ is not as high as in the 47 Tuc case. In addition, we cannot completely exclude that the distance to GLIMPSE-C01 has been overestimated because this key parameter is very difficult to determine in a heavily absorbed case such as this. Despite these problems, the possibility of GLIMPSE-C01 being a Fermi gamma-ray source appears a plausible one when considering all the parameter uncertainties that we have mentioned.

To provide a distance-independent indicator of the emission mechanism, it is instructive to compare the X-ray source counts in the GLIMPSE-C01 and 47 Tuc case. The cluster population of $\mathrm{X}$-ray binaries are indeed believed to be the direct progenitors of the gamma-ray emitting MSPs (see e.g., Bhattacharya 1996, for a review). Pooley et al. (2007) report 13 sources with unabsorbed $0.5-8 \mathrm{keV} X$-ray luminosity above $10^{31} \mathrm{erg} \mathrm{s}^{-1}$. In contrast, the comprehensive X-ray survey of 47 Tuc by Heinke et al. (2005) yielded nearly 3 times more sources above a similar luminosity and energy range. Thus, although Pooley et al. (2007) infer a high production rate of X-ray binary systems by means of close stellar encounters, this is not observationally translated into a significantly enhanced X-ray source population.

Given the evolutionary connection between X-ray binaries and MSPs, the cluster X-ray luminosity is believed to roughly scale to the total number of MSPs. We have therefore computed the cluster $\mathrm{X}$-ray to gamma-ray luminosity ratio according to $L_{0.3-8 \mathrm{kev}} / L_{0.1-1 \mathrm{GeV}}$ based on the observational data quoted above. The resulting value is $\sim 10^{-4}$ for 47 Tuc and $\sim 10^{-5}$ for GLIMPSE-C01. That this ratio is lower by at least an order of magnitude in GLIMPSE-C01 would seem to go against its identification with the Fermi source. The total number of MSP in 47 Tuc is estimated to be $\sim 50$ (Bogdanov et al. 2006; Abdo et al. 2009b). Thus, scaling with the $\mathrm{X}$-ray source luminosity one would expect a lower value of $\sim 20$ in the GLIMPSE C01 case. Nevertheless, we cannot exclude a similar gamma-ray production mechanism being present in both clusters that provides a clear gamma-ray detection with different luminosities in future more sensitive observations.

Alternative scenarios to the one discussed above for GC gamma-ray emission can also be considered. In particular, we cannot exclude other emission mechanisms being at work inside the $\mathrm{GC}$, such as an intermediate-mass black hole at its centre, or peculiar LMXBs. Gamma-ray variability would be expected in this context, although no evidence has been obtained until now.

\subsection{A possible PWN as a counterpart?}

We have also explored the possibility that the Fermi source is associated with another peculiar object inside its $95 \%$ confidence radius. One of them, uncatalogued in the SIMBAD database, is almost at the centre of the Fermi error box with an apparent bubble-like shape, as already mentioned. Its angular diameter extends about $2^{\prime}$ as illustrated in the GLIMPSE image of Fig. 1. 
Table 1. X-ray sources with point-like infrared counterparts inside the 0FGL J1848.6-0138 error circle

\begin{tabular}{cccccccccc}
\hline \hline $\begin{array}{c}\text { 2XMM } \\
\text { source name }\end{array}$ & $\begin{array}{c}\text { Energy flux } \\
(0.5-4.5 \mathrm{keV}) \\
10^{-15} \mathrm{erg} \mathrm{s}^{-1} \mathrm{~cm}^{-2}\end{array}$ & $\begin{array}{c}\text { X-ray/IR } \\
\text { offset }\end{array}$ & $J$ & $H$ & $K \mathrm{~s}$ & $3.6 \mu \mathrm{m}$ & $4.5 \mu \mathrm{m}$ & $5.8 \mu \mathrm{m}$ \\
& & $\mathrm{mag}$ & $\mathrm{mag}$ & $\mathrm{mag}$ & $\mathrm{mag}$ & $\mathrm{mag}$ & $\mathrm{mag}$ \\
\hline $\mathrm{J} 184852.3-014026$ & $26 \pm 4$ & 2.4 & $7.77 \pm 0.02$ & $7.21 \pm 0.05$ & $6.97 \pm 0.03$ & $6.92 \pm 0.04$ & $6.95 \pm 0.04$ & $6.89 \pm 0.03$ \\
$\mathrm{~J} 184813.2-014427$ & $7.6 \pm 2.9$ & 1.8 & $\geq 16.61$ & $14.75 \pm 0.08$ & $12.99 \pm 0.04$ & $11.76 \pm 0.05$ & $11.49 \pm 0.07$ & $11.22 \pm 0.11$ \\
$\mathrm{~J} 184805.0-013726$ & $5.8 \pm 1.4$ & 1.8 & $\geq 16.65$ & $\geq 15.19$ & $13.08 \pm 0.05$ & $10.59 \pm 0.06$ & $9.76 \pm 0.07$ & $9.28 \pm 0.05$ \\
\hline
\end{tabular}

This object is also detected reliably in the radio NVSS images with a $20 \mathrm{~cm}$ integrated flux density of $88 \pm 4 \mathrm{mJy}$, and its morphology is reminiscent of a PWN. Radio emission from this bubble feature is shown in detail in the Fig. 1 right panel but no $\mathrm{X}$-ray detection is obtained when inspecting XMM archival data. The resulting X-ray flux upper limit $(3-\sigma)$ in the $0.5-4.5 \mathrm{keV}$ band is estimated to be $6 \times 10^{-14} \mathrm{erg} \mathrm{s}^{-1} \mathrm{~cm}^{-2}$ for the region covered by the putative PWN. The lack of X-ray detection is difficult to reconcile with a PWN interpretation unless we are dealing with an old, evolved pulsar that has already deposited all its spindown power into the nebula (de Jager et al. 2009).

As an alternative possibility, a newly discovered bubble blown by a central star could also be considered. The stellarlike object closest to the shell centre that we propose to the most likely source of excitation in the shell-like structure is located at RA $=18^{\mathrm{h}} 48^{\mathrm{m}} 43^{\mathrm{s}} .72$ and Dec $=-01^{\circ} 38^{\prime} 38^{\prime \prime} .1$ with $K \mathrm{~s}=13.21 \mathrm{mag}$. Its colours in the 2 Micron All Sky Survey (2MASS) are indicative of a very reddened $\operatorname{star}(J-K \mathrm{~s}=+4.3)$. In this case, we speculate about a possible hadronic interaction in the shocked region of the gas shell that would require further attention.

\subsection{An ultracompact HII region in the field}

Another remarkable object inside the Fermi error circle is the bright radio source GPSR5 31.243-0.110, which likely to be an ultracompact HII region (Giveon et al. 2007) based on its morphology. Its gamma-ray emitting nature is unclear given the lack of suitable physical scenarios for this kind of object.

\subsection{X-ray emitting stellar-like objects in the field}

Several stellar-like objects with X-ray counterparts are also present inside the Fermi error circle as indicated by the comparison between the GLIMPSE and XMM catalogues shown in Fig. 1. None of them is an NVSS radio source. Their observational properties are listed in Table 1 . We cannot exclude any of these stellar-like objects being behind the gamma-ray source, taking into account that a significant fraction of the Fermi sources in the Galactic plane could be related to pulsars both isolated and inside binary systems.

\section{Conclusions}

We have presented an extensive search for counterparts to the unidentified source OFGL J1848.6-0138. As a result, we find what could be the second Fermi gamma-ray source with a possible association with a GC. The emission level observed by Fermi is not perfectly explained by previous theoretical models based on leptons accelerated by the MSP population inside a GC and comptonizing the stellar and microwave background radiation. However, the disagreement between current theories and observation is within an order of magnitude, and does not rule out that a consistent physical scenario is conceivable by means of this physical mechanism. Improved theoretical models and more reliable estimates of the cluster physical parameters (especially the distance) will be required to resolve these apparent discrepancies and, perhaps, confirm the idea that GCs could be gammaray sources.

In addition to the GC scenario, several other peculiar objects inside the Fermi error circle were identified as alternative counterpart candidates, the most interesting of which is very close to the circle centre and resembles a PWN in both infrared and radio images. However, the lack of obvious X-ray emission makes its true nature unclear. Alternatively, it could also be a more ordinary stellar, wind-blown bubble.

Future Fermi observations will certainly reduce the position uncertainty of the gamma-ray source thus enabling us to exclude or confirm some of the counterpart candidates reported here.

Acknowledgements. The authors acknowledge support by grant AYA200768034-C03-02 from the Spanish government, and FEDER funds. This has been also supported by Plan Andaluz de Investigación of Junta de Andalucía as research group FQM322. J.A.C. is a research member of the Consejo Nacional de Investigaciones Científicas y Tecnológicas (CONICET), Argentina. The NRAO is a facility of the NSF operated under cooperative agreement by Associated Universities, Inc. This research made use of the SIMBAD database, operated at the CDS, Strasbourg, France. This publication makes use of data products from the Two Micron All Sky Survey, which is a joint project of the University of Massachusetts and the Infrared Processing and Analysis Center/California Institute of Technology, funded by the National Aeronautics and Space Administration and the National Science Foundation in the USA. We also thank an anonymous referee for helping us to significantly improve this paper.

\section{References}

Abdo. A. A.. Ackermann. M., Ajello. M.. et al. 2009a. ApJS, 183, 46 Abdo. A. A.. Ackermann, M., A jello. M., et al. 2009b. Science. 325. 845 Atwood, W. B., Abdo, A. A., Ackermann, M., et al. 2009, ApJ, 697, 1071 Bednarek. W., \& Sitarek, J. 2007, MNRAS, 377, 920

Benjamin, R. A., Churchwell, E., Babler, B. L., et al. 2003, PASP, 115, 953 Bhattacharya. D. 1996. ASP Conf. Ser.. 105. 547

Bogdanov, S., Grindlay, J. E.. Heinke, C. O., et al. 2006, ApJ, 646, 1104 Chen, K. 1991, Nature, 352, 695

Condon, J. J., Cotton, W. D., Greisen. E. W., et al. 1998, AJ, 115, 1693 de Jager, O. C., et al. 2009, Proc. of the 31th ICRC, Lódz

[arXiv:0906.2644v1]

Giveon, U., Richter, M. J., Becker, R. H., \& White, R. L. 2007, AJ, 133, 639 Heinke, C. O., Grindlay, J. E., Edmonds, P. D., et al. 2005, ApJ, 625, 796 Kobulnicky, H. A., Monson, A. J., Buckalew, B. A., et al. 2005, AJ, 129, 239 Pooley, D.. et al. 2007 [arXiv: 0708 .3365v1]

Tavani, M. 1993, ApJ, 407, 135 